\title{
Nitric oxide-dependent vasodilation induced by minoxidil in isolated rat aorta
}

\author{
Soo Hee Lee ${ }^{1,2, *}$, Seong-Ho Ok ${ }^{2,3,4, *}$, Dawon Kang 5 , Hyun-Jin Kim ${ }^{6,7}$, Seung Hyun Ahn ${ }^{1}$, Sung \\ Il Bae ${ }^{1}$, Ji-Yoon Kim ${ }^{1}$, Eun-Jin Kim ${ }^{5}$, Sunmin Kim ${ }^{1}$, Yeran Hwang ${ }^{1}$ and Ju-Tae Sohn ${ }^{4,8}$ \\ ${ }^{1}$ Department of Anesthesiology and Pain Medicine, Gyeongsang National University Hospital, Jinju-si, Gyeongsangnam-do, \\ Republic of Korea \\ ${ }^{2}$ Department of Anesthesiology and Pain Medicine, Gyeongsang National University Changwon Hospital 11, Samjeongja-ro, \\ Seongsan-gu, Changwon-si, Gyeongsangnam-do, Republic of Korea \\ ${ }^{3}$ Department of Anesthesiology and Pain Medicine, Gyeongsang National University College of Medicine, Jinju-si, \\ Gyeongsangnam-do, Republic of Korea \\ ${ }^{4}$ Institute of Health Sciences, Gyeongsang National University, Jinju-si, Republic of Korea \\ ${ }^{5}$ Department of Physiology, Gyeongsang National University College of Medicine, Jinju-si, Gyeongsangnam-do, Republic of \\ Korea \\ ${ }^{6}$ Division of Applied Life Sciences (BK21 plus), Gyeongsang National University, Gyeongsang, Republic of Korea \\ ${ }^{7}$ Department of Food Science and Technology, Institute of Agriculture and Life Science, Gyeongsang National University, \\ Gyeongsang, Republic of Korea \\ ${ }^{8}$ Department of Anesthesiology and Pain Medicine, Gyeongsang National University College of Medicine, Gyeongsang \\ National University Hospital, Jinju-si, Gyeongsangnam-do, Republic of Korea
}

\begin{abstract}
We examined the effect of endothelium and lipid emulsion on vasodilation induced by minoxidil at a toxic dose and determined the underlying mechanism. The effects of endothelial denudation, $\mathrm{N}^{\mathrm{W}}$-nitro-L-arginine methyl ester (L-NAME), methylene blue, $1 \mathrm{H}$-[1,2,4] oxadiazolo[4,3-a] quinoxalin-1-one (ODQ), and glibenclamide, alone or in combination, on minoxidil-induced vasodilation in endothelium-intact rat aorta were examined. Additionally, the effects of lipid emulsion on minoxidil-induced membrane hyperpolarization and minoxidil concentration were examined. The vasodilatory effects of minoxidil at the toxic dose were higher in endothelium-intact aorta than in endothelium-denuded aorta. L-NAME, methylene blue, ODQ, and glibenclamide attenuated minoxidil-induced vasodilation of endothelium-intact rat aorta. Combined treatment with L-NAME and glibenclamide almost eliminated minoxidil-induced vasodilation. However, lipid emulsion pretreatment did not significantly alter minoxidil-induced vasodilation. Lipid emulsion did not significantly alter minoxidil-induced membrane hyperpolarization and minoxidil concentration. Overall, minoxidil-induced vasodilation is mediated by ATP-sensitive potassium channels and pathways involving nitric oxide and guanylate cyclase.
\end{abstract}

Key words: Minoxidil — Lipid emulsion — Vasodilation — Nitric oxide — Toxic dose

Abbreviations: GC, guanylate cyclase; L-NAME, $\mathrm{N}^{\mathrm{W}}$-nitro-L-arginine methyl ester; NOS, nitric oxide synthase; ODQ, 1H-[1,2,4] oxadiazolo[4,3-a] quinoxalin-1-one; VSMC, vascular smooth muscle cell.

Electronic supplementary material. The online version of this article (doi: 10.4149/gpb_2021012) contains Supplementary material.

\footnotetext{
* These authors contributed equally to this work.

Correspondence to: Ju-Tae Sohn, Department of Anesthesiology and Pain Medicine, Gyeongsang National University Hospital, 79 Gangnam-ro, Jinju-si, 52727, Republic of Korea

E-mail: jtsohn@gnu.ac.kr
} 


\section{Introduction}

Minoxidil induces vasodilation via the activation of ATPsensitive potassium $\left(\mathrm{K}_{\mathrm{ATP}}\right)$ channels (Jahangir and Terzic 2005). A drug with a similar mechanism of vasodilation to minoxidil is levcromakalim. Levcromakalim induces vasodilation via the activation of $\mathrm{K}_{\mathrm{ATP}}$ channels of vascular smooth muscle and partially by endogenous nitric oxide (NO)-induced activation of $\mathrm{K}_{\mathrm{ATP}}$ channels. This suggests that endothelial NO additively contributes to vasodilation mediated by levcromakalim, an $\mathrm{K}_{\mathrm{ATP}}$ channel agonist (Kinoshita et al. 1999; Flagg et al. 2010; Lee et al. 2020). In an in vivo study, nitro-L-arginine, a nitric oxide synthase (NOS) inhibitor, inhibited minoxidil-induced vasodilation of cerebral arterioles (Kontos and Wei 1996). However, other in vitro studies have suggested that minoxidil-induced vasodilation is endothelium independent (Spokas et al. 1983; Meisheri et al. 1988). Hence, the role of endothelial NO on minoxidil-induced vasodilation seems controversial (Spokas et al. 1983; Meisheri et al. 1988; Kontos and Wei 1996; Kinoshita et al. 1999; Lee et al. 2020). Therefore, we investigated whether minoxidil-induced vasodilation is mediated by endothelial NO.

Minoxidil was initially developed to treat hypertension, and it is currently used to treat baldness (Davies et al. 2005). Accidental ingestion of minoxidil at toxic doses, supplied as a topically applied solution to stimulate hair growth, causes severe hypotension and ST-depression (Garrard et al. 2011; Claudet et al. 2015; Gheshlaghi et al. 2018). Vasopressor alpha- 1 adrenoceptor agonists and intravenous fluids are used to treat severe hypotension induced by minoxidil at toxic doses (Garrard et al. 2011; Claudet et al. 2015; Gheshlaghi et al. 2018). Recently, it was reported that lipid emulsion is helpful in treating hypotension caused by the ingestion of minoxidil at toxic doses (Jordan et al. 2018). Lipid emulsions have been used to treat cardiovascular collapse induced by local anesthetic systemic toxicity (Ok et al. 2018). In addition, they have been reported to be effective in treating severe cardiovascular collapse, induced by highly lipid soluble drugs at toxic doses (Ok et al. 2018). The widely accepted underlying mechanism of lipid emulsion treatment is lipid shuttle, suggesting that lipid emulsions absorb highly lipid soluble drugs such as bupivacaine (lipid sink) from vital organ (heart and brain), and subsequently are transported to the liver and muscle, leading to enhanced redistribution (Ok et al. 2018). However, in terms of lipid solubility (log [octanol/water partition coefficient]), lipid sink remains to be controversial in the treatment of cardiovascular collapse induced by non-local anesthetic drugs at toxic doses with high lipid solubility (Mullins et al. 2020). For example, some cases of toxicity induced by bupropion (log [octanol/water partition coefficient] $=3.6$, which has similar lipid solubility to bupivacaine $(\log$ [octanol/water partition coefficient] $=3.41$ ), have been treated with lipid emulsion; whereas, other cases of bupropion toxicity were unresponsive to lipid emulsion treatment (Livshits et al. 2011; Chhabra et al. 2018; Mullins et al. 2020). Furthermore, although a case report suggests that lipid sink (absorption of minoxidil into the lipid phase of lipid emulsion) may contribute to recovery from severe hypotension caused by minoxidil at toxic doses; however, the effect of lipid emulsion on the vasodilation induced by minoxidil at toxic doses remains unknown (Jordan et al. 2018). Thus, we investigated the effect of lipid emulsion on the vasodilation induced by minoxidil at toxic doses. The aim of this study was to examine the role of endothelium and the effect of lipid emulsion (Intralipid) on the vasodilation induced by minoxidil at a toxic dose and to determine the underlying mechanism.

\section{Materials and Methods}

The experimental protocol was approved by the Institutional Animal Care and Use Committee of Gyeongsang National University. All experimental procedures were performed in compliance with the guideline of the Care and Use of Laboratory Animal of Gyeongsang National University.

\section{Preparation of isolated rat aorta and isometric tension measurement}

Male Sprague-Dawley rats (body weight: 250-300 g) were anesthetized with $100 \%$ carbon dioxide, administered via a small hole in the rat cage. Isolated rat aorta was used for measuring isometric tension; it was prepared as described previously (Lee et al. 2020). The rat thoracic cavity was exposed and the descending thoracic rat aorta of each rat was removed from the thoracic cavity and immersed in Krebs solution, composed of $\mathrm{NaCl}(118 \mathrm{mM}), \mathrm{NaHCO}_{3}(25 \mathrm{mM})$, glucose (11 mM), $\mathrm{KCl}(4.7 \mathrm{mM}), \mathrm{CaCl}_{2}(2.4 \mathrm{mM}), \mathrm{MgSO}_{4}$ $(1.2 \mathrm{mM})$, and $\mathrm{KH}_{2} \mathrm{PO}_{4}(1.2 \mathrm{mM})$. The periaortic tissue, which included connective tissue and fat surrounding the isolated descending thoracic aorta (immersed in Krebs solution), was removed under the microscope. The descending thoracic aorta was cut into $2.5-\mathrm{mm}$ long isolated aortic rings. The endothelia of a few aorta were removed by inserting two 25-gauge needles into the aortic lumen and rolling the isolated aorta backward and forward. The isolated thoracic aortae were suspended in a Grass isometric transducer (FT03; Grass Instrument, Massachusetts, USA), installed in the organ bath filled with $10 \mathrm{ml}$ of Krebs solution maintained at $37^{\circ} \mathrm{C}$. Baseline resting tension was maintained at $2.5 \times g$ for $120 \mathrm{~min}$ to reach equilibrium (Klöss et al. 2000). The $\mathrm{pH}$ of the Krebs solution was maintained at 7.4 by aerating it with $95 \%$ oxygen and $5 \%$ carbon dioxide. The integrity of endothelium was confirmed by first adding phenylephrine 
$\left(10^{-7} \mathrm{M}\right)$ to induce a sustained and stable contraction in endothelium-intact aorta. This was followed by the addition of acetylcholine $\left(10^{-5} \mathrm{M}\right)$ to the organ bath. An aorta showing more than $85 \%$ acetylcholine-induced relaxation was regarded as endothelium-intact aorta. In addition, phenylephrine $\left(10^{-8} \mathrm{M}\right)$ induced a sustained and stable contraction of endothelium-denuded aorta. Subsequently, acetylcholine $\left(10^{-5} \mathrm{M}\right)$ was added to the organ bath, and aorta showing less than $15 \%$ acetylcholine-induced relaxation was regarded as endothelium-denuded aorta. The endothelium-intact and -denuded aortae, showing acetylcholine-induced relaxation from phenylephrine-induced contraction, were washed several times with fresh Krebs solution to restore baseline resting tension. Thereafter, the following experimental protocols were performed.

\section{Experimental protocols}

First, the effect of endothelial denudation on minoxidilinduced vasodilation in the isolated rat aorta was examined. Phenylephrine $\left(10^{-6} \mathrm{M}\right)$ was added to the organ bath to produce a stable and sustained contraction of isolated endothelium-intact and -denuded rat aorta. Thereafter, minoxidil $\left(10^{-8}\right.$ to $\left.10^{-4} \mathrm{M}\right)$ was cumulatively added to the organ bath to produce minoxidil concentration-response curves of endothelium-intact and-denuded rat aortae.

Second, we examined the effect of inhibitors of signal pathway, associated with endothelial NO-induced vasodilation, on minoxidil-induced vasodilation in endotheliumintact rat aorta. First, the endothelium-intact aortae were pretreated with the NOS inhibitor $\mathrm{N}^{\mathrm{W}}$-nitro-L-arginine methyl ester (L-NAME, $10^{-4} \mathrm{M}$ ), non-specific guanylate cyclase (GC) inhibitor methylene blue $\left(10^{-6} \mathrm{M}\right)$, and NO-sensitive GC inhibitor $1 \mathrm{H}-[1,2,4]$ oxadiazolo[4,3-a] quinoxalin-1-one (ODQ, $10^{-5} \mathrm{M}$ ) for $15 \mathrm{~min}$ (Lee et al. 2020). Subsequently, phenylephrine $\left(10^{-6} \mathrm{M}\right)$ was added to the organ bath to produce a sustained and stable contraction. Subsequently, minoxidil $\left(10^{-8}\right.$ to $\left.10^{-4} \mathrm{M}\right)$ was cumulatively added to the organ bath to produce minoxidil concentration-response curves in endothelium-intact rat aorta, in the presence or absence of each inhibitor (L-NAME, ODQ, and methylene blue).

Third, the effect of combined treatment with $\mathrm{K}_{\mathrm{ATP}}$ channel inhibitor (glibenclamide) and NOS inhibitor (L-NAME) or glibenclamide alone on minoxidil-induced vasodilation in endothelium-intact rat aorta was examined. After pretreating endothelium-intact aortae with glibenclamide $\left(5 \times 10^{-6} \mathrm{M}\right)$ for $15 \mathrm{~min}$, and then with L-NAME $\left(10^{-4} \mathrm{M}\right)$ for $10 \mathrm{~min}$ or glibenclamide $\left(5 \times 10^{-6} \mathrm{M}\right)$ alone for $25 \mathrm{~min}$, phenylephrine $\left(10^{-6} \mathrm{M}\right)$ was added to the organ bath to produce a stable and sustained contraction (Lee et al. 2020). Subsequently, minoxidil $\left(10^{-8}\right.$ to $\left.10^{-4} \mathrm{M}\right)$ was cumulatively added to the organ bath to produce minoxidil concentration- response curves of the isolated endothelium-intact aorta, in the presence or absence of glibenclamide, alone or in combination with glibenclamide and L-NAME.

Fourth, the effect of pretreatment with lipid emulsion (Intralipid) on minoxidil-induced vasodilation in isolated endothelium-intact and-denuded rat aortae was examined. After pretreating isolated rat aorta with lipid emulsion $(0.5$ and $2 \%$ ) for $20 \mathrm{~min}$, phenylephrine $\left(10^{-6} \mathrm{M}\right)$ was added to the organ bath to produce a stable and sustained contraction in the isolated rat aorta. Thereafter, minoxidil $\left(10^{-8}\right.$ to $10^{-4} \mathrm{M}$ ) was cumulatively added to the organ bath to produce minoxidil concentration-response curves of the isolated endothelium-intact and-denuded rat aorta, in the presence or absence of lipid emulsion. In addition, the effect of post-treatment with lipid emulsion on minoxidil $\left(10^{-4}\right.$ M)-induced vasodilation of isolated endothelium-intact rat aorta was examined. After the induction of sustained and stable contraction of isolated endothelium-intact aorta with phenylephrine $\left(10^{-6} \mathrm{M}\right)$, minoxidil $\left(10^{-4} \mathrm{M}\right)$ was added into organ bath to produce vasodilation. After minoxidil produced a stable and sustained vasodilation, lipid emulsion (0.5 and $2 \%$ ) was added into the organ bath, and subsequently minoxidil $\left(10^{-4} \mathrm{M}\right)$-induced vasodilation was observed for $70 \mathrm{~min}$ in the presence or absence of lipid emulsion.

\section{Cell culture}

Vascular smooth muscle cells (VSMCs), isolated from rat thoracic aortae, were cultured in Dulbecco's modified Eagle's medium (Gibco, Life Technologies, Grand Island, NY, USA), supplemented with $10 \%$ fetal bovine serum (American Type Culture Collection, Manassas, VA, USA), $100 \mathrm{IU} / \mathrm{ml}$ penicillin, and $100 \mu \mathrm{g} / \mathrm{ml}$ streptomycin (Gibco), as previously described (Lee et al. 2019). The cells were maintained in a humidified atmosphere of $5 \% \mathrm{CO}_{2}$ at $37^{\circ} \mathrm{C}$. The cells were grown until they reached sub-confluence, and were then detached with trypsin. For the experiments, the cells were used between passages 3 and 5 .

\section{Recording resting membrane potential of VSMCs}

The membrane potential of VSMCs cultured on poly-Llysine-coated glass coverslips under current clamp mode $(I=0)$ was recorded, using a whole-cell patch clamp technique, as described previously (Baik et al. 2016). The membrane potential was amplified with a patch clamp amplifier (Axopatch 200B; Axon Instruments, Union City, CA, USA). Experimental solutions contained the following: 135 $\mathrm{mM} \mathrm{NaCl}, 5 \mathrm{mM} \mathrm{KCl}, 1 \mathrm{mM} \mathrm{CaCl} 2,1 \mathrm{mM} \mathrm{MgCl}_{2}, 5 \mathrm{mM}$ glucose, $10 \mathrm{mM}$ HEPES as bath solution; and $150 \mathrm{mM} \mathrm{KCl}$, $1 \mathrm{mM} \mathrm{MgCl}_{2}$, $5 \mathrm{mM}$ EGTA, $10 \mathrm{mM}$ HEPES as pipette solution ( $\mathrm{pH} 7.3$ ). The resistance of the pipette was in the range of 4-6 $\mathrm{M} \Omega$. The $\mathrm{pH}$ of the bath and pipette solutions was 
adjusted to 7.4 and 7.3 with $\mathrm{HCl}$ or $\mathrm{KOH}$, respectively. The chemicals were dissolved in the bath solutions at the desired concentrations. All experiments were performed at $25^{\circ} \mathrm{C}$, and the data were analyzed using Clampfit (pCLAMP, version 9.2; Axon Instruments) and OriginPro 2020 (OriginLab Corp., Northampton, MA, USA).

Effect of lipid emulsion on minoxidil concentration in Krebs solution

Minoxidil $\left(10^{-4} \mathrm{M}\right)$ in Krebs solution was mixed with Intralipid $(0.5,2$ and $5 \%)$ using a rotator for $30 \mathrm{~min}$ to emulsify the minoxidil and lipid emulsion, as previously described (Ok et al. 2019a). After centrifugation at 75,000 $\times g$ for $40 \mathrm{~min}$, the minoxidil concentration in the aqueous phase was measured by ultraperformance liquid chromatography-quadrupole time-of-flight mass spectrometry (UPLC-Q-TOF MS; Waters, Milford, MA, USA) with multiple reaction monitoring (MRM). The aqueous layer was injected into an Acquity UPLC BEH $\mathrm{C}_{18}$ column (100 mm $\times 2.1 \mathrm{~mm}, 1.7 \mu \mathrm{m}$; Waters), equilibrated with water/acetonitrile $(95: 5)$ containing $0.1 \%$ formic acid. It was then eluted with a linear gradient (5-100\%) of acetonitrile containing $0.1 \%$ formic acid at a flow rate of $0.35 \mathrm{ml} / \mathrm{min}$ for $7 \mathrm{~min}$. The eluted minoxidil was analyzed by Q-TOF MS (Waters) in the positive electrospray ionization mode with MRM. For analysis using MRM, m/z 289.21 and m/z 140.13 were used as the precursor and product ions of minoxidil, respectively. Capillary and sampling cone voltages were set at $3 \mathrm{kV}$ and $30 \mathrm{~V}$, respectively. The desolvation and source temperatures were $100^{\circ} \mathrm{C}$ and $400^{\circ} \mathrm{C}$, respectively. The desolvation flow rate was set at $800 \mathrm{l} / \mathrm{h}$; LockSpray with leucine-enkephalin $([\mathrm{M}+\mathrm{H}]=556.2771 \mathrm{Da})$ was used at a frequency of $10 \mathrm{~s}$ to ensure reproducibility and accuracy of all analyses. All mass data were collected and analyzed using UIFI 1.8.2 (Waters).

\section{Materials}

Commercially available chemicals of the highest purity were used in this study. Minoxidil, ODQ, L-NAME, glibenclamide, methylene blue, phenylephrine, and acetylcholine were obtained from Sigma Aldrich (St Louis, MO, USA). Intralipid was obtained from Fresenius Kabi AB (Kraftvägen, Kungsängen, Sweden). Glibenclamide and ODQ were dissolved in dimethyl sulfoxide (final concentration of dimethyl sulfoxide $<0.1 \%$ ). Minoxidil was dissolved in ethanol (final concentration: approximately $0.1 \%$ ). All other drugs were dissolved in distilled water.

\section{Statistical analysis}

Data are shown as mean \pm SD and expressed as the percentage of phenylephrine-induced maximal contraction. The effects of endothelial denudation, various inhibitors, and lipid emulsion on vasodilation induced by minoxidil at the toxic dose were analyzed using a generalized linear mixed effect model (Stat version 14.2; StataCorp LP, Lakeway Drive College Station, TX, USA) (Lavergne et al. 2008). The effects of minoxidil, lipid emulsion, and glibenclamide, alone or in combination, on the membrane potential were analyzed using the one-way analysis of variance with post hoc comparisons using Tukey's test (OriginPro 2020). The effect of lipid emulsion on minoxidil concentration in Krebs solution was analyzed using Kruskal-Wallis test, followed by Dunn's multiple comparison test. Results with a $p$ value of less than 0.05 were considered statistically significant.

\section{Results}

Minoxidil-induced vasodilation was higher in the endothelium-intact aorta than in the endothelium-denuded rat aorta (Figs. 1 and $2 ; p<0.05 v s$. endothelium-denuded at $3 \times 10^{-7}$
A

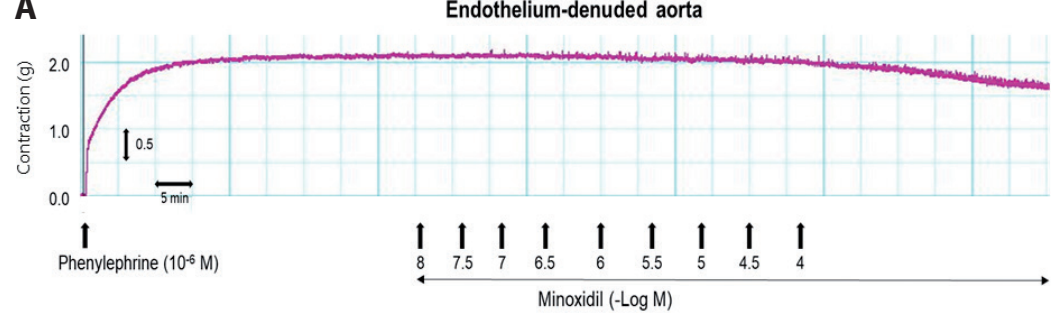

B

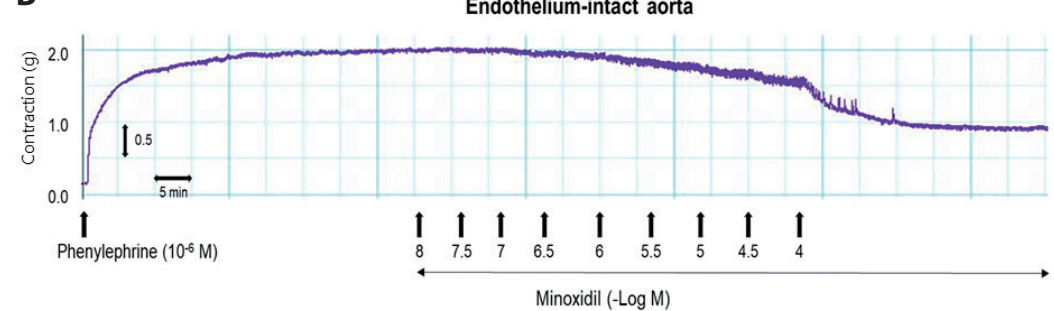

Figure 1. Concentration $\left(10^{-8}\right.$ to $\left.10^{-4} \mathrm{M}\right)$ response curves of minoxidil traces in endothelium-denuded (A) and endothelium-intact (B) rat aortae precontracted with phenylephrine $\left(10^{-6} \mathrm{M}\right)$. 
to $\left.10^{-4} \mathrm{M}\right)$. The NOS inhibitor L-NAME $\left(10^{-4} \mathrm{M}\right)$ attenuated minoxidil-induced vasodilation of the endothelium-intact rat aorta (Fig. 3A and supplementary Fig. S1; $p<0.05 v s$. control at $3 \times 10^{-7}$ to $\left.10^{-4} \mathrm{M}\right)$. The non-specific GC inhibitor methylene blue $\left(10^{-6} \mathrm{M}\right)$ and NO-sensitive GC inhibitor ODQ $\left(10^{-5} \mathrm{M}\right)$ inhibited minoxidil-induced vasodilation of the isolated endothelium-intact rat aorta (Fig. 3B and C, Figs. S2 and S3; $p<0.01$ vs. control at $10^{-6}$ to $\left.10^{-4} \mathrm{M}\right)$. Glibenclamide $\left(5 \times 10^{-6} \mathrm{M}\right)$ inhibited minoxidil-induced vasodilation of the endothelium-intact rat aorta (Fig. 4; $p<0.01$ vs. control at $3 \times 10^{-5}$ and $\left.10^{-4} \mathrm{M}\right)$. The combined treatment with glibenclamide $\left(5 \times 10^{-6} \mathrm{M}\right)$ and L-NAME $\left(10^{-4} \mathrm{M}\right)$ had a more pronounced inhibitory effect on minoxidil-induced vasodilation than glibenclamide alone $\left(5 \times 10^{-6} \mathrm{M}\right)$ in the endothelium-intact rat aorta (Fig. $4 ; p<0.01 v s$. glibenclamide alone at $10^{-6}$ to $\left.10^{-4} \mathrm{M}\right)$. Pretreatment with lipid emulsion (0.5 and 2\%) did not significantly alter minoxidilinduced vasodilation of endothelium-intact rat aorta (Fig. $5 \mathrm{~A})$. In addition, pretreatment with lipid emulsion ( 0.5 and $2 \%$ ) had no effect on minoxidil-induced vasodilation of the endothelium-denuded aorta in rats (Fig. 5B). However, post-treatment with lipid emulsion ( 0.5 and $2 \%)$ transiently increased minoxidil $\left(10^{-4} \mathrm{M}\right)$-induced vasodilation (Fig. $5 \mathrm{C}$; $p<0.05 v s$. time-matched control at $10 \mathrm{~min}$ ) of endotheliumintact aorta slightly at 10 and 20 min after lipid emulsion administration. Thereafter, the post-treatment with lipid emulsion ( 0.5 and $2 \%$ ) did not significantly alter minoxidil $\left(10^{-4} \mathrm{M}\right)$-induced vasodilation compared with that in the time-matched control group (Fig. 5C) of endothelium-intact aorta at 30 to $70 \mathrm{~min}$ after lipid emulsion administration. Minoxidil $\left(10^{-4} \mathrm{M}\right)$ induced membrane hyperpolarization (Fig. 6; $p<0.05$ vs. control). However, lipid emulsion $(0.5$ and $2 \%$ ) did not significantly alter minoxidil-induced membrane hyperpolarization (Fig. 6). Glibenclamide $\left(10^{-5} \mathrm{M}\right)$ inhibited minoxidil $\left(10^{-4} \mathrm{M}\right)$-induced membrane hyper-

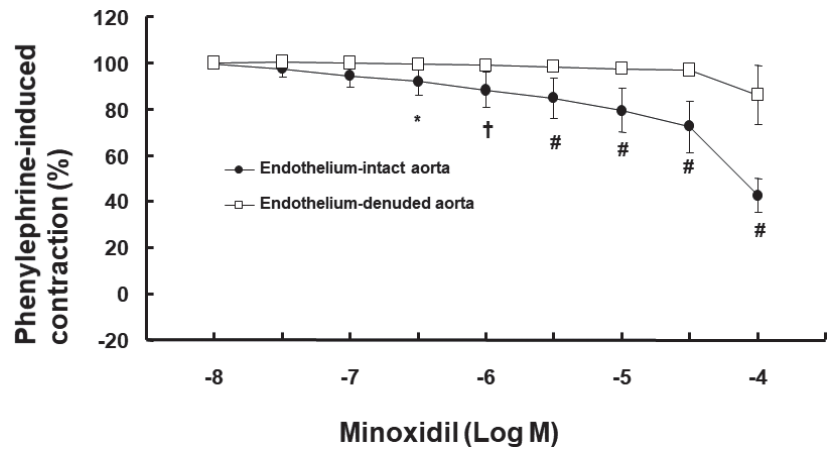

Figure 2. Effect of endothelial denudation on vasodilation induced by minoxidil in isolated rat aorta. Data $(n=6)$ are shown as mean $\pm \mathrm{SD}$ and expressed as a percentage of phenylephrine $\left(10^{-6} \mathrm{M}\right)$ induced contractions. $n$ indicates the number of rats from which the descending thoracic aortae were obtained. ${ }^{\star} \mathrm{p}<0.05,{ }^{\dagger} p<0.01$, and ${ }^{\#} p<0.001 v s$. endothelium-denuded rat aortae.

polarization (minoxidil alone: $-48.8 \pm 1.9 \mathrm{mV} v s$. minoxidil plus glibenclamide: $-41.6 \pm 1.4 \mathrm{mV} ; p<0.001 v s$. minoxidil alone). Moreover, lipid emulsion (0.5, 2 and 5\%) did not significantly alter minoxidil concentration $\left(10^{-4} \mathrm{M}\right)$ in Krebs solution (Fig. 7).

\section{Discussion}

The results of this study suggest that minoxidil-induced vasodilation of isolated rat aorta, precontracted with phenylephrine, is mediated by $\mathrm{K}_{\mathrm{ATP}}$ channels and endothelial NO release. In addition, it was observed that lipid emulsion did not significantly alter minoxidil-induced vasodilation. The major findings of this study are as follows: 1) minoxidilinduced vasodilation was present in endothelium-intact
A

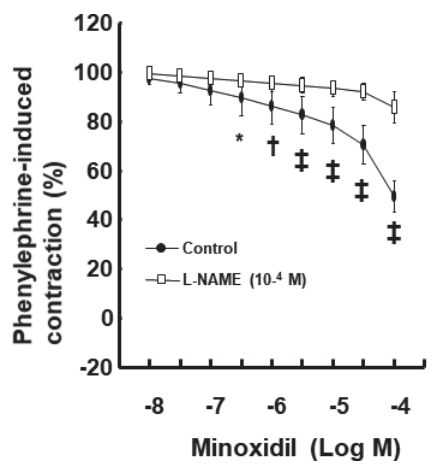

B

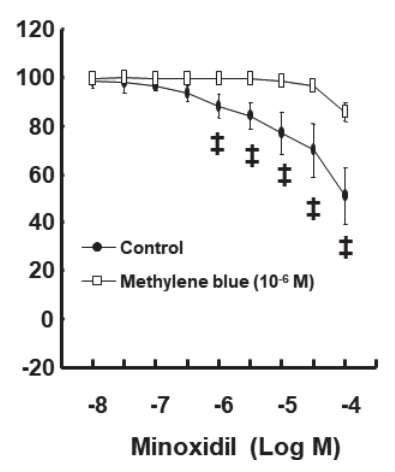

C

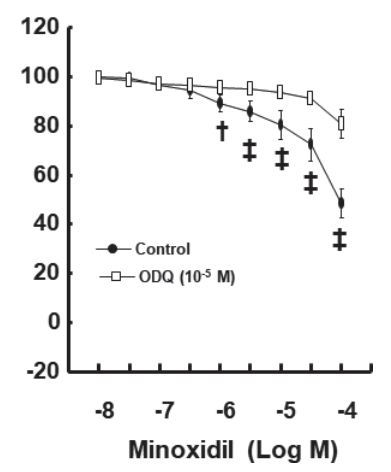

Figure 3. Effect of $\mathrm{N}^{\mathrm{W}}$-nitro-L-arginine methyl ester (L-NAME; A), methylene blue (B), and 1H-[1,2,4] oxadiazolo[4,3-a]quinoxalin-1-one (ODQ; C) on vasodilation induced by minoxidil in endothelium-intact rat aorta. Data $(n=6)$ are shown as mean \pm SD and expressed as a percentage of phenylephrine $\left(10^{-6} \mathrm{M}\right)$-induced contractions. $n$ indicates the number of rats from which the descending thoracic aortae were obtained. ${ }^{*} p<0.05,{ }^{\dagger} p<0.01$, and ${ }^{\ddagger} p<0.001 v$ s. control. 


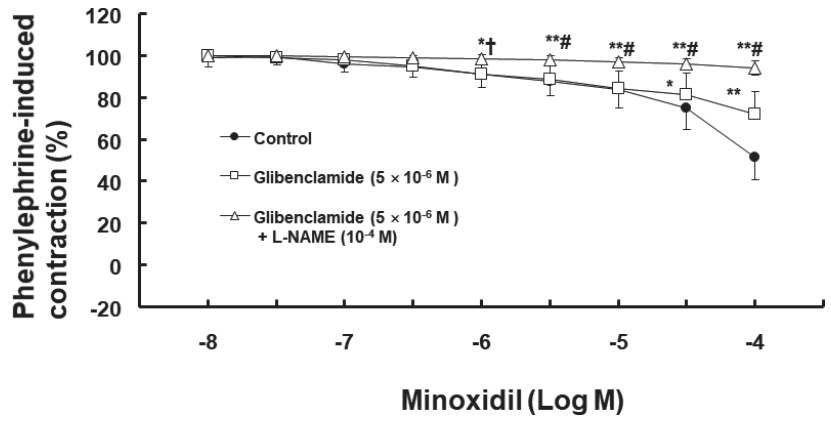

Figure 4. Effects of glibenclamide alone or $\mathrm{N}^{\mathrm{W}}$-nitro-L-arginine methyl ester (L-NAME) plus glibenclamide combined treatment on vasodilation induced by minoxidil in endothelium-intact rat aorta. Data $(n=11)$ are shown as mean \pm SD and expressed as a percentage of phenylephrine $\left(10^{-6} \mathrm{M}\right)$-induced contractions. $n$ indicates the number of isolated rat aorta. ${ }^{*} \mathrm{p}<0.01$ and ${ }^{* *} \mathrm{p}<$ $0.001 v$ s. control. ${ }^{\dagger} p<0.01$ and ${ }^{\#} p<0.001 v s$. glibenclamide alone.

rat aorta, 2) L-NAME attenuated vasodilation induced by minoxidil in endothelium-intact rat aorta, and 3) lipid emulsion did not significantly alter vasodilation and membrane hyperpolarization induced by minoxidil at the toxic dose.

The vasodilatory effect of the $\mathrm{K}_{\mathrm{ATP}}$ channel agonist, minoxidil, was more pronounced in endothelium-intact aorta than in endothelium-denuded aorta (Figs. 1 and 2) (Brayden 2002; Jahangir and Terzic 2005). In addition, studies have shown that endothelial NO contributes to vasodilation induced by the $\mathrm{K}_{\mathrm{ATP}}$ channel agonist levcromakalim (Kinoshita et al. 1999; Flagg et al. 2010; Lee et al. 2020). Thus, we investigated the effect of inhibitors of the cellular pathway, which is associated with vasodilation induced by endothelial NO, on minoxidil-induced vasodilation in endothelium-intact rat aorta. Similar to the findings of previous studies on levcromakalim, NOS inhibitor LNAME, NO-sensitive GC inhibitor ODQ, and non-specific GC inhibitor methylene blue inhibited minoxidil-induced vasodilation, suggesting that minoxidil-induced vasodilation is partially mediated by the endothelial NO-GC pathway (Kinoshita et al. 1999; Lee et al. 2020). In a previous study, minoxidil $\left(10^{-3} \mathrm{M}\right)$-induced vasodilation of endotheliumintact rat aorta, precontracted with serotonin, was $77 \pm 4 \%$ (Kauffman et al. 1986). In contrast, in this study, minoxidil $\left(10^{-4} \mathrm{M}\right)$-induced maximal vasodilation of endotheliumintact rat aorta, precontracted with phenylephrine, was 57 $\pm 4 \%$. In addition, in a previous study, methylene blue did not significantly alter minoxidil-induced vasodilation of endothelium-intact rat aorta, whereas methylene blue in the current study attenuated minoxidil $\left(10^{-4} \mathrm{M}\right)$-induced maximal vasodilation (Kauffman et al. 1986). Furthermore, it has also been reported that minoxidil $\left(6 \times 10^{-8}\right.$ to $\left.6 \times 10^{-6} \mathrm{M}\right)$ induced vasodilation of isolated rabbit aorta is endothelium independent and minoxidil sulfate induces vasodilation of rabbit mesenteric artery in an endothelium-independent manner (Spokas et al. 1983; Meisheri et al. 1988). While it is difficult to explain this difference in findings between the current study and previous studies, the following factors could be attributed to the difference (Spokas et al. 1983; Kauffman et al. 1986; Meisheri et al. 1988): 1) the use of different maximal concentrations of minoxidil $\left(10^{-4} \mathrm{M} v s\right.$. $10^{-3} \mathrm{M}$ ), 2) use of different species (rat vs. rabbit), 3) use of different locations (aorta vs. mesenteric artery), 4) use of different preconstrictors (phenylephrine vs. serotonin or norepinephrine), 5) use of different compounds (parent compound: minoxidil $v s$. its metabolite: minoxidil sulfate), and 6) use of different forms of isolated rat aorta (aortic ring $v s$. aortic strip). The NOS inhibitor nitro-L-arginine eliminated minoxidil-induced vasodilation in pial arteriole in anesthetized rats, suggesting that minoxidil-induced vasodilation may involve NO release (Kontos and Wei 1996). Consistent with minoxidil $\left(10^{-4} \mathrm{M}\right)$-induced vasodilation of isolated endothelium-denuded rat aorta in the current study, minoxidil $\left(10^{-4} \mathrm{M}\right)$ caused membrane hyperpolarization of rat

A

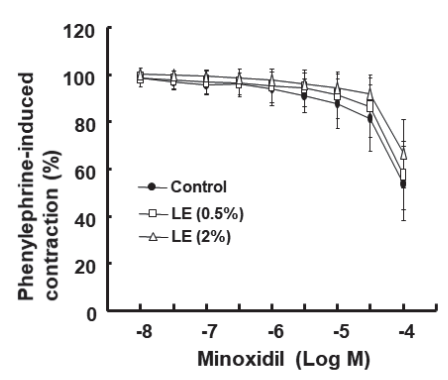

B

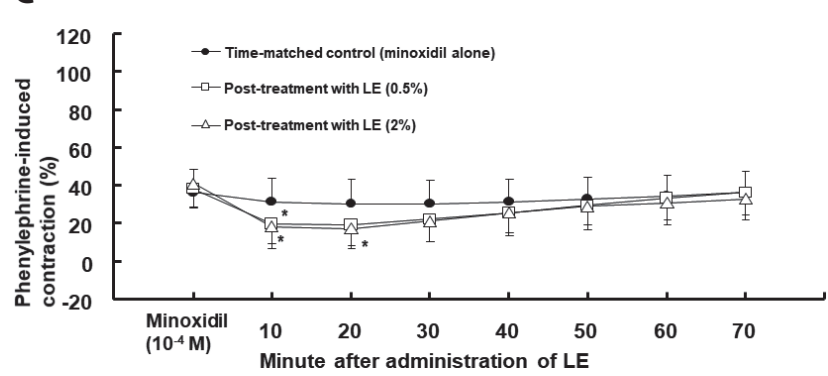

Figure 5. Effect of pretreatment with lipid emulsion (LE) on minoxidil-induced vasodilation in endothelium-intact $(\mathbf{A}, n=6)$ and endothelium-denuded (B, $n=5)$ rat aortae. Data are shown as mean \pm SD and expressed as a percentage of phenylephrine $\left(10^{-6}\right.$ $\mathrm{M}$ )-induced contractions. $n$ indicates the number of rats from which the descending thoracic aortae were obtained. C. Effect of post-treatment with lipid emulsion (LE) on minoxidil $\left(10^{-4}\right.$ $\mathrm{M})$-induced vasodilation in endothelium-intact rat aortae. Data $(n=10)$ are shown as mean \pm SD and expressed as a percentage of phenylephrine $\left(10^{-6} \mathrm{M}\right)$-induced contractions. $n$ indicates the number of descending thoracic aortae. ${ }^{\star} p<0.05 v s$. timematched control. 
aortic VSMCs (Fig. 6). $\mathrm{K}_{\mathrm{ATP}}$ channels in the vascular smooth muscle consist of four inward rectifying potassium channel subunits (Kir6.1 or Kir6.2) and four regulatory sulfonylurea receptors (SUR2B), which are regulated by ATP/ADP and inhibited by glibenclamide (Brayden 2002; Jahangir and Terzic 2005). Minoxidil is expected to have two binding sites in the C-terminus of SUR (Schwanstecher et al. 1998). In addition to the site to which P1075 binds, SUR2B has another distinct site for minoxidil binding (Schwanstecher et al. 1998). Minoxidil has a greater binding affinity to SUR2 than to the SUR1 isoform in the presence of MgATP (Schwanstecher et al. 1998), suggesting that minoxidil induces vasodilation through SUR2B. In line with the findings of a previous study, the $\mathrm{K}_{\mathrm{ATP}}$ channel inhibitor glibenclamide inhibited minoxidil-induced vasodilation of endothelium-intact rat aorta (Fig. 4) and minoxidil-induced membrane hyperpolarization. This suggests that minoxidil-induced vasodilation is mediated by the $\mathrm{K}_{\mathrm{ATP}}$ channels (Wickenden et al. 1991). In addition, combined treatment with glibenclamide and the NOS inhibitor L-NAME significantly reduced minoxidil $\left(10^{-4} \mathrm{M}\right)$-induced maximal vasodilation (Fig. 4), suggesting that endogenous NO contributes to minoxidil-induced vasodilation. Further research to examine the cellular signal pathway associated with minoxidil-induced NO-mediated vasodilation is needed.

Lipid emulsion is effective in treating intractable cardiovascular collapse induced by drugs with high lipid solubility, including calcium channel blockers, at toxic doses (Ok et al. 2018). Severe hypotension and tachycardia induced by accidental oral administration of minoxidil at a toxic dose (topical solution form) in adults and children can be treated with intravenous fluids and vasopressors, including alpha-1 adrenoceptor agonists (midodrine and phenylephrine), dopamine, and norepinephrine (Farrell and Epstein 1999; Aprahamian et al. 2011; Garrard et al. 2011; Claudet et al. 2015; Gheshlaghi et al. 2018). In addition, it has been reported that hypotension and tachycardia induced by the ingestion of minoxidil at toxic doses can be successfully treated with intravenous fluid, dopamine, and esmolol, followed by intravenous administration of lipid emulsion (Jordan et al. 2018). Although minoxidil is relatively less lipid soluble (log [octanol/water partition coefficient] = 1.24), a case report suggested that lipid emulsion may sequester minoxidil into the lipid phase of lipid emulsion (Jordan et al. 2018; minoxidil). However, in the present study, pretreatment with Intralipid (2\%), which is more than the lipid emulsion concentration (1\%) required to exert scavenging effect and inotropic effect in the treatment of drug toxicity induced via oral administration, did not significantly alter vasodilation induced by minoxidil at toxic doses (Fettiplace et al. 2015). Unexpectedly, the post-treatment with lipid emulsion transiently and slightly enhanced minoxidil (toxic concentration)-induced vasodilation for $20 \mathrm{~min}$ (Fig. 5C).

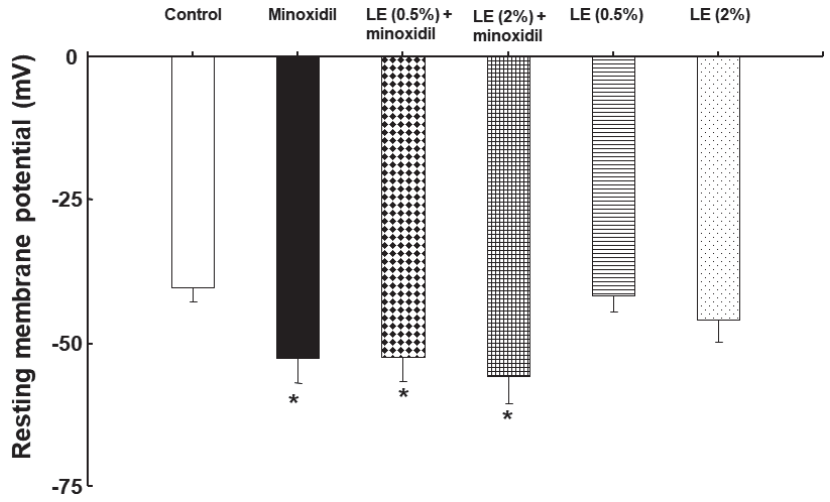

Figure 6. Effect of minoxidil $\left(10^{-4} \mathrm{M}\right)$ and lipid emulsion (LE), alone or in combination, on membrane hyperpolarization in rat aortic vascular smooth muscle cells. Membrane potentials were recorded within $1 \mathrm{~min}$ after treatment with chemicals under the current clamp mode $(I=0)$. Each bar represents the mean $\pm S D$ of eight repeated experiments. The values correspond to three independent experiments. ${ }^{\star} p<0.001 v$ s. control.

Thereafter, it did not significantly alter minoxidil-induced vasodilation (Fig. 5C). Furthermore, consistent with the results of isometric tension measurement, Intralipid did not significantly alter minoxidil-induced membrane hyperpolarization of rat aortic VSMCs (Fig. 6). It has been reported that the HULC-Q-TOF method to measure drug concentration in Krebs solution treated with lipid emulsion partitioned highly lipid soluble drugs (bupivacaine, verapamil, and amlodipine) into the lipid phase of lipid emulsion (Lee et al. 2018; Ok et al. 2019a, 2019b). Consistent with the above-mentioned results of the current study, Intralipid ( 0.5 , 2 and 5\%) did not significantly alter minoxidil concentration (Fig. 7). Taken together, these results, (including the results

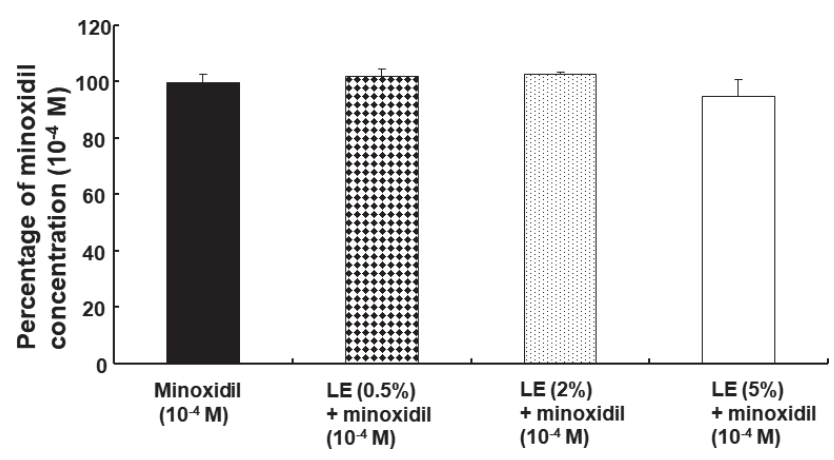

Figure 7. Effect of lipid emulsion (LE) on minoxidil concentration $\left(10^{-4} \mathrm{M}\right)$ in Krebs solution. Minoxidil concentration was measured by ultraperformance liquid chromatography-quadrupole time-offlight mass spectrometry with positive electrospray ionization and multiple reaction monitoring mode. Experiments were repeated three times. Data are shown as mean \pm SD and expressed as a percentage of minoxidil concentration $\left(10^{-4} \mathrm{M}\right)$. 
of high-performance liquid chromatography) suggest that the lipid sink theory, in which lipid emulsion absorbs highly lipid soluble drugs, such as bupivacaine, verapamil, amlodipine, and flecainide, into the lipid phase, was not applicable in the present study (Weinberg 2012). This difference in the findings between the case report and current in vitro study can be attributed to the following factors (Jordan et al. 2018). First, after the initial administration of dopamine and esmolol for the treatment of minoxidil-induced hypotension and tachycardia, lipid emulsion was administered intravenously. Thus, the recovery from minoxidil toxicity may be due to prior administration of dopamine and esmolol. Second, while a highly lipid soluble drug has more than 2 log octanol/water partition coefficient, the log octanol/water partition coefficient of minoxidil is 1.24 , which indicates its relatively low lipid solubility (Weinberg 2012; minoxidil). In addition, the magnitude of reduction in the concentration of each drug with different lipid solubility by lipid emulsion is correlated with the lipid solubility of each drug (French et al. 2011). Overall, the results of this study, in conjunction with the findings of previous studies, suggest that lipid emulsion may have no significant beneficial effect in treating severe hypotension induced by minoxidil toxicity (French et al. 2011; Weinberg 2012). Third, as minoxidil at toxic doses induces cardiac toxicity including myocardial ischemia, whereas lipid emulsion attenuates myocardial ischemic damage, this lipid emulsion-induced recovery observed in the case report may be associated with the cardioprotective effective (Hanton et al. 2008; Umar et al. 2018). Thus, further research is needed to examine both effect of lipid emulsion on hemodynamic depression induced by minoxidil at toxic doses and the underlying mechanism, which post-treatment with lipid emulsion transiently increased minoxidil-induced vasodilation.

The present study had a few limitations that need to be considered while interpreting the results. Peripheral vascular resistance, which contributes to blood pressure, is mainly determined by small resistance arterioles (Clifford 2011). However, we used the aorta as a conduit vessel in the current experiment. Minoxidil sulfate, the active metabolite of minoxidil, produced via multiple sulfotransferases, mainly contributes to decreased blood pressure and hair growth (Buhl et al. 1990; Kikuchi et al. 2016). However, in the current study, the parent compound minoxidil, was used. Despite these limitations, the results of this study suggest that minoxidil-induced vasodilation may be attenuated in patients with compromised endothelial function. In addition, from the results of the tension study, electrophysiological study, and high-performance liquid chromatography, it was evident that lipid emulsion may not reverse vasodilation induced by minoxidil at a toxic dose $\left(10^{-4} \mathrm{M}\right)$ exceeding approximately the toxic serum concentration (approximately $8 \times 10^{-8} \mathrm{M}$ ) of minoxidil (Mesfin et al. 1996).
In conclusion, these results suggest that minoxidilinduced vasodilation in endothelium-intact rat aorta is mediated by $\mathrm{K}_{\mathrm{ATP}}$ channels and the pathways involving $\mathrm{NO}$ and GC. In addition, lipid emulsion did not significantly alter minoxidil (toxic dose)-induced vasodilation in isolated rat aorta. This suggests that lipid emulsion has no therapeutic effect on severe hypotension due to vasodilation induced by minoxidil at toxic doses.

Conflict of interest. The authors declared that there is no conflict of interest.

Acknowledgement. This research was supported by a grant from the Institute of Health Sciences (lHS GNU-2020-02).

\section{References}

Aprahamian A, Escoda S, Patteau G, Merckx A, Chéron G (2011): Minoxidil intoxication, the pharmacological agent of a hair lotion. Arch. Pediatr. 18, 1302-1304 (in French) https://doi.org/10.1016/j.arcped.2011.08.031

Baik J, Ok SH, Kim EJ, Kang D, Hong JM, Shin IW, Lee HK, Chung YK, Cho Y, Lee SH, et al. (2016): Mepivacaine attenuates vasodilation induced by ATP-sensitive potassium channels in rat aorta. Can. J. Physiol. Pharmacol. 94, 1211-1219 https://doi.org/10.1139/cjpp-2016-0041

Brayden JE (2002): Functional roles of KATP channels in vascular smooth muscle. Clin. Exp. Pharmacol. Physiol. 29, 312-316 https://doi.org/10.1046/j.1440-1681.2002.03650.x

Buhl AE, Waldon DJ, Baker CA, Johnson GA (1990): Minoxidil sulfate is the active metabolite that stimulates hair follicles. J. Invest. Dermatol. 95, 553-557 https://doi.org/10.1111/1523-1747.ep12504905

Chhabra N, DesLauriers C, Wahl M, Bryant SM (2018): Management of severe bupropion poisoning with intravenous lipid emulsion. Clin. Toxicol. (Phila) 56, 51-54 https://doi.org/10.1080/15563650.2017.1337909

Claudet I, Cortey C, Honorat R, Franchitto N (2015): Minoxidil topical solution: an unsafe product for children. Pediatr. Emerg. Care 31, 44-46 https://doi.org/10.1097/PEC.0000000000000301

Clifford PS (2011): Local control of blood flow. Adv. Physiol. Educ. 35, 5-15

https://doi.org/10.1152/advan.00074.2010

Davies GC, Thornton MJ, Jenner TJ, Chen YJ, Hansen JB, Carr RD, Randall VA (2005): Novel and established potassium channel openers stimulate hair growth in vitro: implications for their modes of action in hair follicles. J. Invest. Dermatol. 124, 686-694 https://doi.org/10.1111/j.0022-202X.2005.23643.x

Farrell SE, Epstein SK (1999): Overdose of rogaine extra strength for men topical minoxidil preparation. J. Toxicol. Clin. Toxicol. 37, 781-783 https://doi.org/10.1081/CLT-100102457

Fettiplace MR, Akpa BS, Rubinstein I, Weinberg G (2015): Confusion about infusion: rational volume limits for intravenous 
lipid emulsion during treatment of oral overdoses. Ann. Emerg. Med. 66, 185-188

https://doi.org/10.1016/j.annemergmed.2015.01.020

Flagg TP, Enkvetchakul D, Koster JC, Nichols CG (2010): Muscle KATP channels: recent insights to energy sensing and myoprotection. Physiol. Rev. 90, 799-829 https://doi.org/10.1152/physrev.00027.2009

French D, Smollin C, Ruan W, Wong A, Drasner K, Wu AH (2011): Partition constant and volume of distribution as predictors of clinical efficacy of lipid rescue for toxicological emergencies. Clin. Toxicol. (Phila) 49, 801-809 https://doi.org/10.3109/15563650.2011.617308

Garrard A, Wood A, Sollee D, Aaronson P (2011): Refractory hypotension due to Rogaine ${ }^{\circledast}$ (minoxidil) ingestion managed with midodrine. Clin. Toxicol. (Phila) 49, 907-909 https://doi.org/10.3109/15563650.2011.624988

Gheshlaghi F, Zoofaghari S, Dorooshi G (2018): Unstable angina: a rare presentation of minoxidil intoxication: a case report and literature review. J. Res. Pharm. Pract. 7, 210-212 https://doi.org/10.4103/jrpp.JRPP_18_23

Hanton G, Sobry C, Daguès N, Rochefort GY, Bonnet P, Eder V (2008): Cardiovascular toxicity of minoxidil in the marmoset. Toxicol. Lett. 180, 157-165 https://doi.org/10.1016/j.toxlet.2008.05.018

Jahangir A, Terzic A (2005): K(ATP) channel therapeutics at the bedside. J. Mol. Cell. Cardiol. 39, 99-112 https://doi.org/10.1016/j.yjmcc.2005.04.006

Jordan TJM, Yaxley PE, Culler CA, Balakrishnan A (2018): Successful management of minoxidil toxicosis in a dog. J. Am. Vet. Med. Assoc. 252, 222-226 https://doi.org/10.2460/javma.252.2.222

Kauffman RF, Schenck KW, Conery BG, Cohen ML (1986): Effects of pinacidil on serotonin-induced contractions and cyclic nucleotide levels in isolated rat aortae: comparison with nitroglycerin, minoxidil, and hydralazine. J. Cardiovasc. Pharmacol. 8, 1195-200 https://doi.org/10.1097/00005344-198611000-00015

Kikuchi S, Fujita Y, Onodera M, Fujino Y, Inoue Y (2016): Prolonged hypotension induced by ingesting a topical minoxidil solution: analysis of minoxidil and its metabolites. Acute. Med. Surg. 3, 384-387 https://doi.org/10.1002/ams2.196

Kinoshita H, Iwahashi S, Kakutani T, Mizumoto K, Iranami H, Hatano Y (1999): The role of endothelium-derived nitric oxide in relaxations to levcromakalim in the rat aorta. Jpn. J. Pharmacol. 81, 362-366 https://doi.org/10.1254/jjp.81.362

Klöss S, Bouloumié A, Mülsch A (2000): Aging and chronic hypertension decrease expression of rat aortic soluble guanylyl cyclase. Hypertension 35, 43-47 https://doi.org/10.1161/01.HYP.35.1.43

Kontos HA, Wei EP (1996): Arginine analogues inhibit responses mediated by ATP-sensitive K+ channels. Am. J. Physiol. 271, H1498-H1506 https://doi.org/10.1152/ajpheart.1996.271.4.H1498

Lavergne C, Martinez M, Trottier C (2008): Empirical model selection in generalized linear mixed effects models. Computational. Statistics 23, 99-109 https://doi.org/10.1007/s00180-007-0071-y
Lee SH, Kang D, Ok SH, Kwon SC, Kim HJ, Kim EJ, Hong JM, Kim JY, Bae SI, An S, Sohn JT (2018): Linoleic acid attenuates the toxic dose of bupivacaine-mediated reduction of vasodilation evoked by the activation of adenosine triphosphate-sensitive potassium channels. Int. J. Mol. Sci. 2018, 19 https://doi.org/10.3390/ijms19071876

Lee SH, Kwon SC, Ok SH, Hong JM, Kim JY, Ahn SH, Il Bae S, Shin Y, Sohn JT (2019): Levobupivacaine-induced vasoconstriction involves caldesmon phosphorylation mediated by tyrosine kinase-induced ERK phosphorylation. Eur. J. Pharmacol. 842, $167-176$ https://doi.org/10.1016/j.ejphar.2018.10.055

Lee SH, Kang D, Ok SH, Kim JY, Bae SI, Hwang Y, Park KE, Kim JW, Sohn JT (2020): Lipofundin MCT/LCT inhibits levcromakaliminduced vasodilation by inhibiting endothelial nitric oxide release. Int. J. Mol. Sci. 21, 1763 https://doi.org/10.3390/ijms21051763

Livshits Z, Feng Q, Chowdhury F, Amdo TD, Nelson LS, Hoffman RS (2011): Life-threatening bupropion ingestion: is there a role for intravenous fat emulsion? Basic. Clin. Pharmacol. Toxicol. 109, 418-422 https://doi.org/10.1111/j.1742-7843.2011.00750.x

Meisheri KD, Cipkus LA, Taylor CJ (1988): Mechanism of action of minoxidil sulfate-induced vasodilation: a role for increased K+ permeability. J. Pharmacol. Exp. Ther. 245, 751-760

Mesfin GM, Higgins MJ, Robinson FG, Zhong WZ (1996): Relationship between serum concentrations, hemodynamic effects, and cardiovascular lesions in dogs treated with minoxidil. Toxicol. Appl. Pharmacol. 140, 337-344 https://doi.org/10.1006/taap.1996.0229

Mullins ME, Seger DL (2020): Antidotal use of lipid emulsion - the pendulum swings. Clin. Toxicol. (Phila) 58, 1281-1283 https://doi.org/10.1080/15563650.2020.1746800

Ok SH, Hong JM, Lee SH, Sohn JT (2018): Lipid emulsion for treating local anesthetic systemic toxicity. Int. J. Med. Sci. 15, 713-722 https://doi.org/10.7150/ijms.22643

Ok SH, Lee SH, Kim JY, Kim HJ, Bae SI, Hwang Y, Tak S, Sohn JT (2019a): Lipid emulsion inhibits the vasodilation induced by a toxic dose of amlodipine in isolated rat aortae. Int. J. Med. Sci. 16, 1621-1630 https://doi.org/10.7150/ijms.38502

Ok SH, Lee SH, Kim HJ, Hong JM, Kim JY, Bae SI, Shin Y, Sohn JT (2019b): Lipid emulsion attenuates the vasodilation induced by a toxic dose of a calcium channel blocker through its partitioning into the lipid phase. Gen. Physiol. Biophys. 38, 227-235 https://doi.org/10.4149/gpb_2019008

Schwanstecher M, Sieverding C, Dörschner H, Gross I, AguilarBryan L, Schwanstecher C, Bryan J (1998): Potassium channel openers require ATP to bind to and act through sulfonylurea receptors. EMBO J. 17, 5529-5535 https://doi.org/10.1093/emboj/17.19.5529

Spokas EG, Folco G, Quilley J, Chander P, McGiff JC (1983): Endothelial mechanism in the vascular action of hydralazine. Hypertension 5, I107- I111 https://doi.org/10.1161/01.HYP.5.2_Pt_2.I107

Umar S, Li J, Hannabass K, Vaillancourt M, Cunningham CM, Moazeni S, Mahajan A, Eghbali M (2018): Free fatty acid 
receptor G-protein-coupled receptor 40 mediates lipid emulsion-induced cardioprotection. Anesthesiology 129, 154-162

https://doi.org/10.1097/ALN.0000000000002195

Weinberg GL (2012): Lipid emulsion infusion: resuscitation for local anesthetic and other drug overdose. Anesthesiology 117, 180-187

https://doi.org/10.1097/ALN.0b013e31825ad8de
Wickenden AD, Grimwood S, Grant TL, Todd MH (1991): Comparison of the effects of the $\mathrm{K}(+)$-channel openers cromakalim and minoxidil sulphate on vascular smooth muscle. Br. J. Pharmacol. 103, 1148-1152

https://doi.org/10.1111/j.1476-5381.1991.tb12315.x

Received: January 5, 2021

Final version accepted: April 5, 2021 


\section{Supplementary Material}

\section{Nitric oxide-dependent vasodilation induced by minoxidil in isolated rat aorta}

Soo Hee Lee ${ }^{1,2, *}$, Seong-Ho Ok ${ }^{2,3,4, *}$, Dawon Kang ${ }^{5}$, Hyun-Jin Kim ${ }^{6,7}$, Seung Hyun Ahn ${ }^{1}$, Sung Il Bae ${ }^{1}$, Ji-Yoon Kim ${ }^{1}$, Eun-Jin Kim ${ }^{5}$, Sunmin Kim ${ }^{1}$, Yeran Hwang ${ }^{1}$ and Ju-Tae Sohn ${ }^{4,8}$

${ }^{1}$ Department of Anesthesiology and Pain Medicine, Gyeongsang National University Hospital, Jinju-si, Gyeongsangnam-do, Republic of Korea

2 Department of Anesthesiology and Pain Medicine, Gyeongsang National University Changwon Hospital 11, Samjeongja-ro, Seongsan-gu, Changwon-si, Gyeongsangnam-do, Republic of Korea

${ }^{3}$ Department of Anesthesiology and Pain Medicine, Gyeongsang National University College of Medicine, Jinju-si, Gyeongsangnam-do, Republic of Korea

${ }^{4}$ Institute of Health Sciences, Gyeongsang National University, Jinju-si, Republic of Korea

${ }^{5}$ Department of Physiology, Gyeongsang National University College of Medicine, Jinju-si, Gyeongsangnam-do, Republic of Korea

${ }^{6}$ Division of Applied Life Sciences (BK21 plus), Gyeongsang National University, Gyeongsang, Republic of Korea

${ }^{7}$ Department of Food Science \& Technology, Institute of Agriculture and Life Science, Gyeongsang National University, Gyeongsang, Republic of Korea

${ }^{8}$ Department of Anesthesiology and Pain Medicine, Gyeongsang National University College of Medicine, Gyeongsang National University Hospital, Jinju-si, Gyeongsangnam-do, Republic of Korea

Supplementary Figures

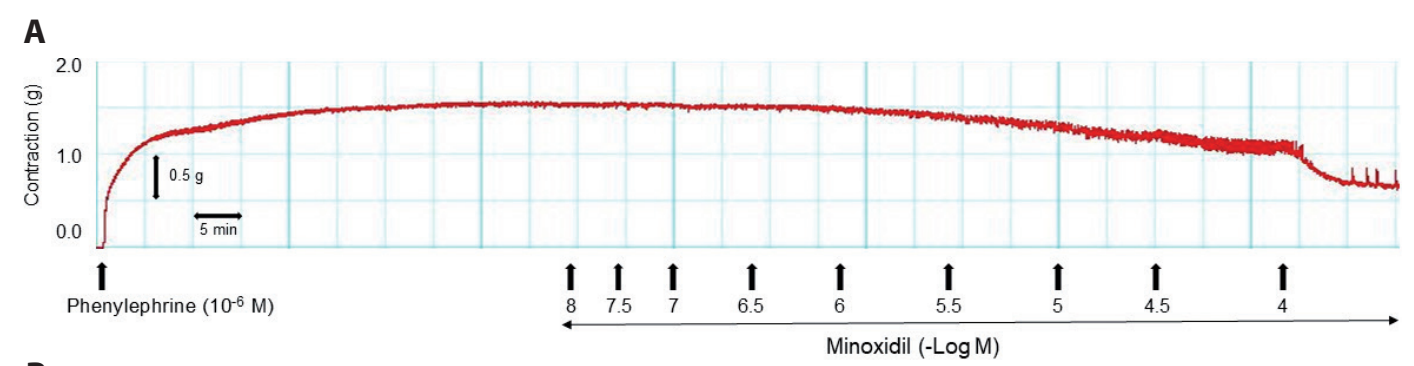

B

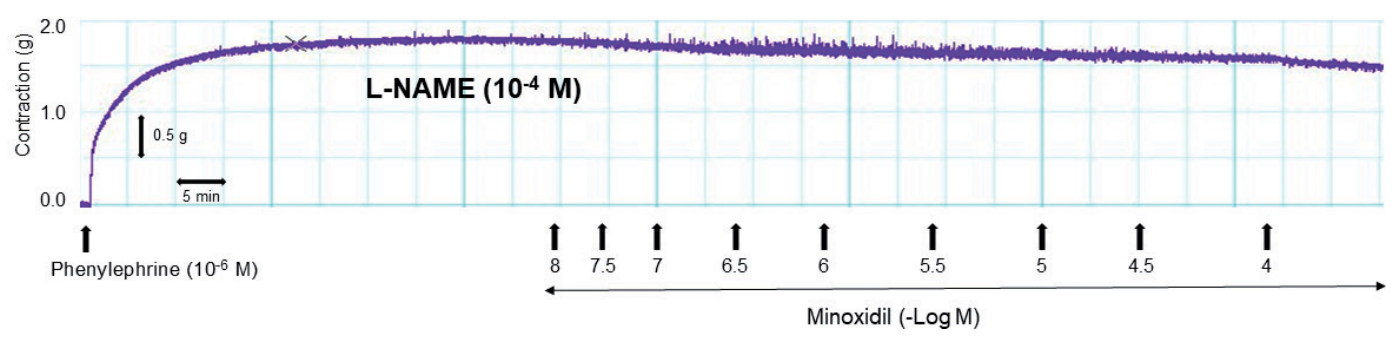

Figure S1. Original recording showing minoxidil concentration $\left(10^{-8}\right.$ to $\left.10^{-4} \mathrm{M}\right)$-response curves of endothelium-intact rat aortae without (A) or with $(\mathbf{B}) \mathrm{N}^{\mathrm{W}}$-nitro-L-arginine methyl ester (L-NAME, $\left.10^{-4} \mathrm{M}\right)$; the aortae were precontracted with phenylephrine $\left(10^{-6} \mathrm{M}\right)$. 


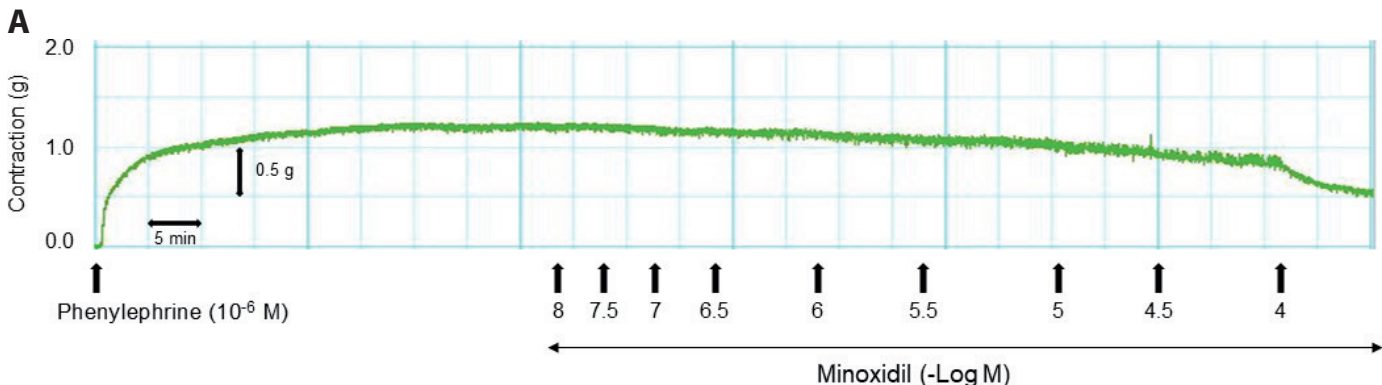

B

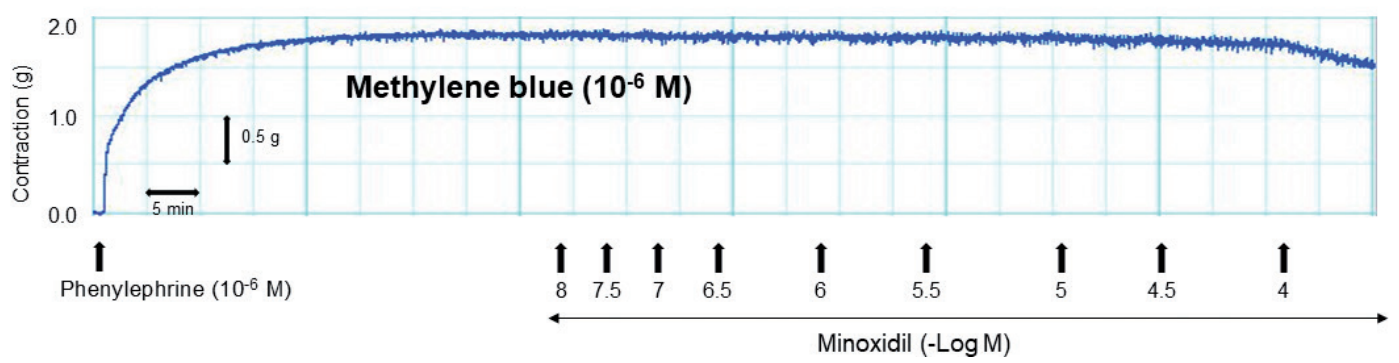

Figure S2. Original recording showing minoxidil concentration $\left(10^{-8}\right.$ to $\left.10^{-4} \mathrm{M}\right)$-response curves of endothelium-intact rat aortae without (A) or with (B) methylene blue $\left(10^{-6} \mathrm{M}\right)$; the aortae were precontracted with phenylephrine $\left(10^{-6} \mathrm{M}\right)$.

A

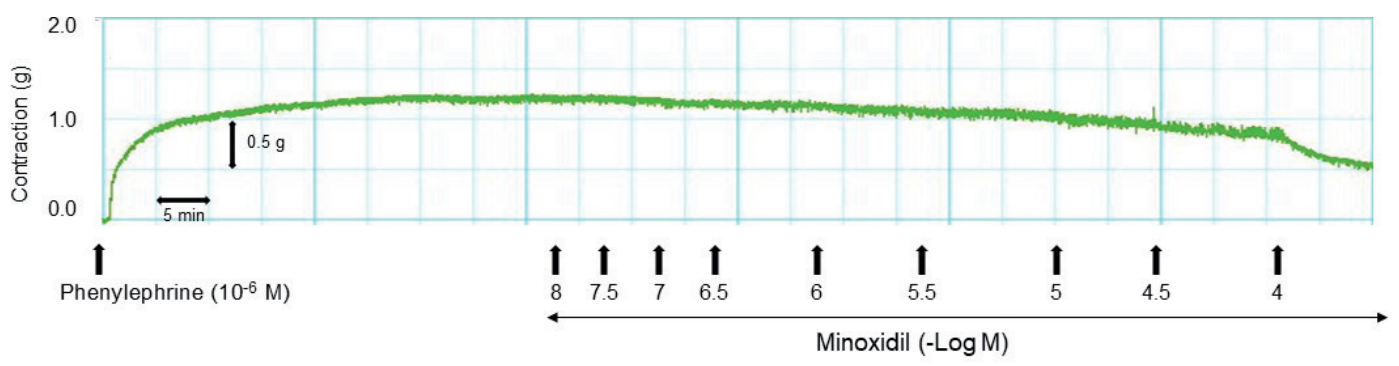

B

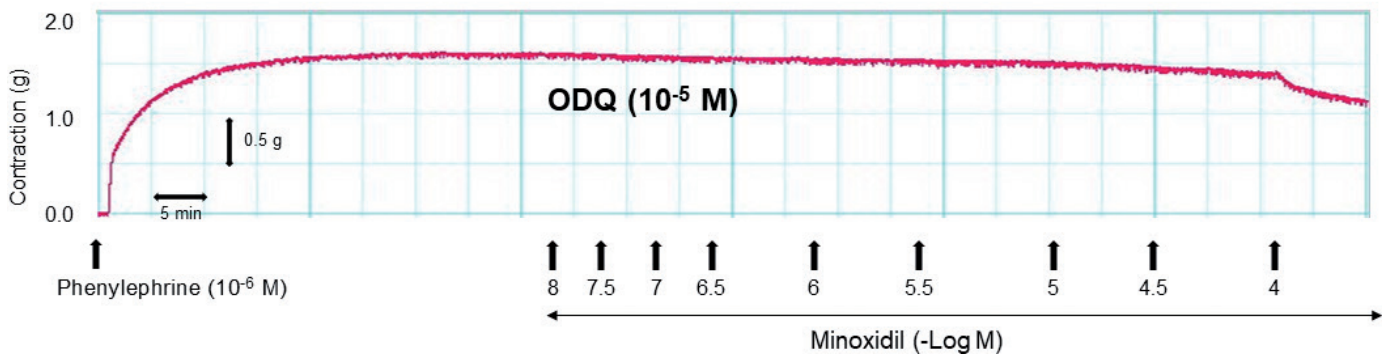

Figure S3. Original recording showing minoxidil concentration $\left(10^{-8}\right.$ to $\left.10^{-4} \mathrm{M}\right)$-response curves of endothelium-intact rat aortae without (A) or with (B) $1 \mathrm{H}$-[1,2,4] oxadiazolo[4,3-a] quinoxalin-1-one (ODQ, $\left.10^{-5} \mathrm{M}\right)$; the aortae were precontracted with phenylephrine $\left(10^{-6} \mathrm{M}\right)$. 